University of Texas at El Paso

ScholarWorks@UTEP

\title{
$5-2018$
}

\section{Why Quantum (Wave Probability) Models Are a Good Description of Many Non-Quantum Complex Systems, and How to Go Beyond Quantum Models}

\author{
Miroslav Svitek \\ Czech Technical University in Prague, svitek@fd.cvut.cz \\ Olga Kosheleva \\ The University of Texas at El Paso, olgak@utep.edu \\ Vladik Kreinovich

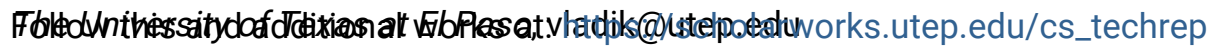 \\ ¿.PCarth. Noguyenputer Sciences Commons \\ Bemkimgedtriversity of Ho Chi Minh City, ajeb@buh.edu.vn
}

Technical Report: UTEP-CS-18-46

Svítek M., Kosheleva O., Kreinovich V., Nguyen T.N. (2019) Why Quantum (Wave Probability) Models Are a Good Description of Many Non-quantum Complex Systems, and How to Go Beyond Quantum Models. In: Kreinovich V., Thach N., Trung N., Van Thanh D. (eds) Beyond Traditional Probabilistic Methods in Economics. ECONVN 2019. Studies in Computational Intelligence, vol 809. Springer, Cham

https://doi.org/10.1007/978-3-030-04200-4_13

\section{Recommended Citation}

Svitek, Miroslav; Kosheleva, Olga; Kreinovich, Vladik; and Nguyen, Thach N., "Why Quantum (Wave Probability) Models Are a Good Description of Many Non-Quantum Complex Systems, and How to Go Beyond Quantum Models" (2018). Departmental Technical Reports (CS). 1236.

https://scholarworks.utep.edu/cs_techrep/1236

This Article is brought to you for free and open access by the Computer Science at ScholarWorks@UTEP. It has been accepted for inclusion in Departmental Technical Reports (CS) by an authorized administrator of ScholarWorks@UTEP. For more information, please contact Iweber@utep.edu. 


\title{
Why Quantum (Wave Probability) Models Are a Good Description of Many Non-Quantum Complex Systems, and How to Go Beyond Quantum Models
}

Miroslav Svítek, Olga Kosheleva, Vladik Kreinovich, and Thach Ngoc Nguyen

\begin{abstract}
In many practical situations, it turns out to be beneficial to use techniques from quantum physics in describing non-quantum complex systems. For example, quantum techniques have been very successful in econometrics and, more generally, in describing phenomena related to human decision making. In this paper, we provide a possible explanation for this empirical success. We also show how to modify quantum formulas to come up with an even more accurate descriptions of the corresponding phenomena.
\end{abstract}

\section{Formulation of the Problem}

Quantum models are often a good description of non-quantum systems: a surprising phenomenon. Quantum physics has been designed to describe quantum objects, i.e., objects - mostly microscopic but sometimes macroscopic as well that exhibit quantum behavior. Somewhat surprisingly, however, it turns out that quantum-type techniques - techniques which are called wave probability techniques in $[16,17]$ - can also be useful in describing non-quantum complex systems, in particular, economic systems and other systems involving human behavior, etc.; see, e.g., $[1,5,9,16,17]$ and references therein.

Miroslav Svítek

Faculty of Transportation Sciences, Czech Technical University in Prague, Konviktska 20

CZ-110 00 Prague 1, Czech Republic, e-mail: svitek@fd.cvut.cz

Olga Kosheleva and Vladik Kreinovich

University of Texas at El Paso, 500 W. University, El Paso, TX 79968, USA

e-mail: olgak@utep.edu,vladik@utep.edu

Thach Ngoc Nguyen

Banking University of Ho Chi Minh City, 56 Hoang Dieu 2, Quan Thu Duc, Thu Duc

Ho Chi Minh City, Vietnam, e-mail: Thachnn@buh.edu.vn 
Why quantum techniques can help in non-quantum situations is largely a mystery.

Natural questions. The first natural question is why? Why quantum models are often a good description of non-quantum systems.

The next natural question is related to the fact that while quantum models provide a good description of non-quantum systems, this description is not perfect. So, a natural question: how to get a better approximation?

What we do in this paper. In this paper, we provide answers to the above two questions.

\section{Towards an Explanation}

Ubiquity of multi-D normal distributions. To describe the state of a complex system, we need to describe the values of the quantities $x_{1}, \ldots, x_{n}$ that form this state.

In many cases, the system consists of a large number of reasonably independent parts. In this case, each of the quantities $x_{i}$ describing the system is approximately equal to the sum of the values of the corresponding quantity that describes these parts. For example:

- The overall trade volume of a country can be described as the sum of the trades performed by all its companies and all its municipal units.

- Similarly, the overall number of unemployed people in a country is equal to the sum of numbers of unemployed folks in different regions, etc.

It is known that the distribution of the sum of a large number of independent random variables is - under certain reasonable conditions - close to Gaussian (normal); this result is known as the Central Limit Theorem; see, e.g., [15].

Thus, with reasonable accuracy, we can assume that the vectors $x=\left(x_{1}, \ldots, x_{n}\right)$ formed by all the quantities that characterize the system as a whole are normally distributed.

Let us simplify the description of the multi-D normal distribution. A multi-D normal distribution is uniquely characterized by its means $\mu=\left(\mu_{1}, \ldots, \mu_{n}\right)$, where $\mu_{i} \stackrel{\text { def }}{=} E\left[x_{i}\right]$, and by its covariance matrix $\sigma_{i j} \stackrel{\text { def }}{=} E\left[\left(x_{i}-\mu_{i}\right) \cdot\left(x_{j}-\mu_{j}\right)\right]$.

By observing the values of the characteristics $x_{i}$ corresponding to different systems, we can estimate the mean values $\mu_{i}$ and thus, instead of the original values $x_{i}$, consider deviations $\delta_{i} \stackrel{\text { def }}{=} x_{i}-\mu_{i}$ from these values.

For these deviations, the description is simpler. Indeed, their means are 0s, so to fully describe the distribution of the corresponding vector $\delta=\left(\delta_{1}, \ldots, \delta_{n}\right)$, it is sufficient to know the covariance matrix $\sigma_{i j}$.

An additional simplification is that since the means are all $0 \mathrm{~s}$, the formula for the covariance matrix has a simplified form $\sigma_{i j}=E\left[\delta_{i} \cdot \delta_{j}\right]$. 
For complex systems, with a large number of parameters, a further simplification is needed. After the above simplification, to fully describe the corresponding distribution, we need to describe all the values of the $n \times n$ covariance matrix $\sigma_{i j}$. In general, an $n \times n$ matrix contains $n^{2}$ elements, but since the covariance matrix is symmetric, we only need to describe

$$
\frac{n \cdot(n+1)}{2}=\frac{n^{2}}{2}+\frac{n}{2}
$$

parameters - slightly more than half as many.

The big question is: can we determine all these parameters from the observations?

In general in statistics, if we want to find a reasonable estimate for a parameter, we need to have a certain number of observations. Based on $N$ observations, we can find the value of each quantity with accuracy $\approx \frac{1}{\sqrt{N}} ;$ see, e.g., [15]. Thus, to be able to determine a parameter with a reasonable accuracy of $20 \%$, we need to select $N$ for which $\frac{1}{\sqrt{N}} \approx 20 \%=0.2$, i.e., $N=25$. So, to find the value of one parameter, we need approximately 25 observations. By the same logic, for any integer $k$, to find the values of $k$ parameters, we need to have $25 k$ observations. In particular, to determine $\frac{n \cdot(n+1)}{2} \approx \frac{n^{2}}{2}$ parameters, we need to have $25 \cdot \frac{n^{2}}{2}$ observations.

Each fully detailed observation of a system leads to $n$ numbers $x_{1}, \ldots, x_{n}$ and thus, to $n$ numbers $\delta_{1}, \ldots, \delta_{n}$. So, to estimate $25 \cdot \frac{n^{2}}{2}=12.5 \cdot n^{2}$ parameters, we need to have $12.5 \cdot n$ different systems. And we often do not have that many system to observe.

For example, to have a detailed analysis of a country's economics, we need to have at least several dozen parameters, at least $n \cdot 30$. By the above logic, to fully describe the joint distribution of all these parameters, we will need at least

$$
12.5 \cdot 30 \approx 375
$$

countries - and on the Earth, we do not have that many of them.

This problem occurs not only in econometrics, it is even more serious, e.g., in medical applications of bioinformatics: there are thousands of genes, and not enough data to be able to determine all the correlations between them.

Since we cannot determine the covariance matrix $\sigma_{i j}$ exactly, we therefore need to come up with an approximate description, a description that would require fewer parameters.

Need for a geometric description. What does it means to have a good approximation? Intuitively, approximations means having a model which is, in some reasonable sense, close to the original one - i.e., is at a small distance from the original model. Thus, to come up with an understanding of what is a good approximation, it is desirable to have a geometric representation of the corresponding problem, a representation in which different objects would be represented by points in a certain 
space - so that we could easily understand what is the distance between different objects.

From this viewpoint, to see how we can reasonably approximate multi-D normal distributions, it is desirable to use an appropriate geometric representation of such distributions. Good news is that such a representation is well known. Let us recall this representation.

Geometric description of multi-D normal distribution: reminder. It is well known that a 1D normally distributed random variable $x$ with 0 mean and standard deviation $\sigma$ can be presented as $\sigma \cdot X$, where $X$ is "standard" normal distribution, with 0 mean and standard deviation 1 .

Similarly, it is known that any normally distributed $n$-dimensional random vector $\delta=\left(\delta_{1}, \ldots, \delta_{n}\right)$ can be represented as linear combinations $\delta_{i}=\sum_{j=1}^{n} a_{i j} \cdot X_{j}$ of $n$ independent standard random variables $X_{1}, \ldots, X_{n}$. These variables can be found, e.g., as eigenvectors of the covariance matrix divided by the corresponding eigenvalues.

This way, each of the original quantities $\delta_{i}$ is represented by the $n$-dimensional vector $a_{i}=\left(a_{i 1}, \ldots, a_{i n}\right)$. The known geometric feature of this representation is that for every two linear combinations $\delta^{\prime}=\sum_{i=1}^{n} c_{i}^{\prime} \cdot \delta_{i}$ and $\delta^{\prime \prime}=\sum_{i=1}^{n} c_{i}^{\prime \prime} \cdot \delta_{i}$ of the quantities $\delta_{i}$ :

- the standard deviation $\sigma\left[\delta^{\prime}-\delta^{\prime \prime}\right]$ of the difference between these linear combinations is equal to

- the (Euclidean) distance $d\left(a^{\prime}, a^{\prime \prime}\right)$ between the corresponding $n$-dimensional vectors $a^{\prime}=\sum_{i=1} c_{i}^{\prime} \cdot a_{i}$ and $a^{\prime \prime}=\sum_{i=1} c_{i}^{\prime \prime} \cdot a_{i}$, with components $a_{j}^{\prime}=\sum_{i=1}^{n} c_{i}^{\prime} \cdot a_{i j}$ and $a_{j}^{\prime \prime}=$ $\sum_{i=1}^{n} c_{i}^{\prime \prime} \cdot a_{i j}$ :

$$
\sigma\left[\delta^{\prime}-\delta^{\prime \prime}\right]=d\left(a^{\prime}, a^{\prime \prime}\right)
$$

Indeed, since $\delta_{i}=\sum_{j=1}^{n} a_{i j} \cdot X_{j}$, we conclude that

$$
\delta^{\prime}=\sum_{i=1}^{n} c_{i}^{\prime} \cdot \delta_{i}=\sum_{i=1}^{n} c_{i}^{\prime} \cdot \sum_{j=1}^{n} a_{i j} \cdot X_{j}
$$

By combining together all the coefficients at $X_{j}$, we conclude that

$$
\delta^{\prime}=\sum_{j=1}^{n}\left(\sum_{i=1}^{n} c_{i}^{\prime} \cdot a_{i j}\right) \cdot X_{j}
$$

i.e., by using the formula for $a_{j}^{\prime}$, that

$$
\delta^{\prime}=\sum_{j=1}^{n} a_{j}^{\prime} \cdot X_{j}
$$


Similarly, we can conclude that

$$
\delta^{\prime \prime}=\sum_{j=1}^{n} a_{j}^{\prime \prime} \cdot X_{j}
$$

thus

$$
\delta^{\prime}-\delta^{\prime \prime}=\sum_{j=1}^{n}\left(a_{j}^{\prime}-a_{j}^{\prime \prime}\right) \cdot X_{j}
$$

Since the mean of the difference $\delta^{\prime}-\delta^{\prime \prime}$ is thus equal to 0 , the square of its standard deviation is simply equal to $\sigma^{2}\left[\delta^{\prime}-\delta^{\prime \prime}\right]=E\left[\left(\delta^{\prime}-\delta^{\prime \prime}\right)^{2}\right]$. In our case,

$$
\left(\delta^{\prime}-\delta^{\prime \prime}\right)^{2}=\sum_{i=1}^{n}\left(a_{j}^{\prime}-a_{j}^{\prime \prime}\right)^{2} \cdot X_{j}^{2}+\sum_{i \neq j}\left(a_{i}^{\prime}-a_{i}^{\prime \prime}\right) \cdot\left(a_{j}^{\prime}-a_{j}^{\prime \prime}\right) \cdot X_{i} \cdot X_{j}
$$

Thus,

$$
\begin{gathered}
\sigma^{2}\left[\delta^{\prime}-\delta^{\prime \prime}\right]=E\left[\left(\delta^{\prime}-\delta^{\prime \prime}\right)^{2}\right]= \\
\sum_{i=1}^{n}\left(a_{j}^{\prime}-a_{j}^{\prime \prime}\right)^{2} \cdot E\left[X_{j}^{2}\right]+\sum_{i \neq j}\left(a_{i}^{\prime}-a_{i}^{\prime \prime}\right) \cdot\left(a_{j}^{\prime}-a_{j}^{\prime \prime}\right) \cdot E\left[X_{i} \cdot X_{j}\right] .
\end{gathered}
$$

The variables $X_{j}$ are independent and have 0 mean, so for $i \neq j$, we have $E\left[X_{i} \cdot X_{j}\right]=$ $E\left[X_{i}\right] \cdot E\left[X_{j}\right]=0$. For each $i$, since $X_{i}$ are standard normal distributions, we have $E\left[X_{j}^{2}\right]=1$. Thus, we conclude that

$$
\sigma^{2}\left[\delta^{\prime}-\delta^{\prime \prime}\right]=\sum_{i=1}^{n}\left(a_{j}^{\prime}-a_{j}^{\prime \prime}\right)^{2}
$$

i.e., indeed, $\sigma^{2}\left[\delta^{\prime}-\delta^{\prime \prime}\right]=d^{2}\left(a^{\prime}, a^{\prime \prime}\right)$ and thus, $\sigma\left[\delta^{\prime}-\delta^{\prime \prime}\right]=d\left(\delta^{\prime}, \delta^{\prime \prime}\right)$.

How can we use this geometric description to find a fewer-parameters $(k \ll n)$ approximation to the corresponding situation. We have $n$ quantities $x_{1}, \ldots, x_{n}$ that describe the complex system. By subtracting the mean values $\mu_{i}$ from each of the quantities, we get shifted values $\delta_{1}, \ldots, \delta_{n}$. To absolutely accurately describe the joint distribution of these $n$ quantities, we need to describe $n n$-dimensional vectors $a_{1}, \ldots, a_{n}$ corresponding to each of these quantities.

In our approximate description, we still want to keep all $n$ quantities, but we cannot keep them as $n$-dimensional vectors - this would require too many parameters to determine, and, as we have mentioned earlier, we do not have that many observations to be able to experimentally determine all these parameters. Thus, the natural thing to do is to decrease their dimension.

In other words:

- instead of representing each quantity $\delta_{i}$ as an $n$-dimensional vector $a_{i}=$ $\left(a_{i 1}, \ldots, a_{i n}\right)$ corresponding to $\delta_{i}=\sum_{j=1}^{n} a_{i j} \cdot X_{j}$ 
- we select some value $k \ll n$ and represent each quantity $\delta_{i}$ as a $k$-dimensional vector $a_{i}=\left(a_{i 1}, \ldots, a_{i k}\right)$ corresponding to $\delta_{i}=\sum_{j=1}^{k} a_{i j} \cdot X_{j}$.

For $k=2$, the above approximation idea leads to a quantum-type description. In one of the simplest cases $k=2$, each quantity $\delta_{i}$ is represented by a 2-D vector $a_{i}=\left(a_{i 1}, a_{i 2}\right)$. Similarly to the above full-dimensional case, for every two linear combinations $\delta^{\prime}=\sum_{i=1}^{n} c_{i}^{\prime} \cdot \delta_{i}$ and $\delta^{\prime \prime}=\sum_{i=1}^{n} c_{i}^{\prime \prime} \cdot \delta_{i}$ of the quantities $\delta_{i}$,

- the standard deviation $\sigma\left[\delta^{\prime}-\delta^{\prime \prime}\right]$ of the difference between these linear combinations is equal to

- the (Euclidean) distance $d\left(a^{\prime}, a^{\prime \prime}\right)$ between the corresponding 2-dimensional vectors $a^{\prime}=\sum_{i=1}^{n} c_{i}^{\prime} \cdot a_{i}$ and $a^{\prime \prime}=\sum_{i=1}^{n} c_{i}^{\prime \prime} \cdot a_{i}$, with components $a_{j}^{\prime}=\sum_{i=1}^{n} c_{i}^{\prime} \cdot a_{i j}$ and $a_{j}^{\prime \prime}=\sum_{i=1}^{n} c_{i}^{\prime \prime} \cdot a_{i j}$ :

$$
\sigma\left[\delta^{\prime}-\delta^{\prime \prime}\right]=d\left(a^{\prime}, a^{\prime \prime}\right)=\sqrt{\left(a_{1}^{\prime}-a_{1}^{\prime \prime}\right)^{2}+\left(a_{2}^{\prime}-a_{2}^{\prime \prime}\right)^{2}}
$$

However, in the 2-D case, we can alternatively represent each 2-D vector $a_{i}=$ $\left(a_{i 1}, a_{i 2}\right)$ as a complex number

$$
a_{i}=a_{i 1}+\mathrm{i} \cdot a_{i 2}
$$

where, as usual, $\mathrm{i} \stackrel{\text { def }}{=} \sqrt{-1}$. In this representation, the modulus (absolute value)

$$
\left|a^{\prime}-a^{\prime \prime}\right|
$$

of the difference

$$
a^{\prime}-a^{\prime \prime}=\left(a_{1}^{\prime}-a_{1}^{\prime \prime}\right)+\mathrm{i} \cdot\left(a_{2}^{\prime}-a_{2}^{\prime \prime}\right)
$$

is equal to $\sqrt{\left(a_{1}^{\prime}-a_{1}^{\prime \prime}\right)^{2}+\left(a_{2}^{\prime}-a_{2}^{\prime \prime}\right)^{2}}$, i.e., exactly the distance between the original points.

Thus, in this approximation:

- each quantity is represented by a complex number, and

- the standard deviation of the difference between different quantities is equal to the modulus of the difference between the corresponding complex numbers - and thus, the variance is equal to the square of this modulus,

- in particular, the standard deviation of each linear combination is equal to the modulus of the corresponding complex number - and thus, the variance is equal to the square of this modulus.

This is exactly what happens when we use quantum-type formulas. Thus, we have indeed explained the empirical success of quantum-type formulas as a reasonable approximation to the description of complex systems. 
Comment. Similar argument explain why, in fuzzy logic (see, e.g., [2, 10, 6, 12, 13, 18]) complex-valued quantum-type techniques have also been successfully used see, e.g., $[4,7,8,11,14]$.

What can we do to get a more accurate description of complex systems? As we have mentioned earlier, while quantum-type descriptions are often reasonably accurate, quantum formulas often do not provide the exact description of the corresponding complex systems. So, how can we extend and/or modify these formulas to get a more accurate description?

Based on the above arguments, a natural way to do is to switch from complexvalued 2-dimensional $(k=2)$ approximate descriptions to higher-dimensional $(k=$ $3, k=4$, etc.) descriptions, where:

- each quantity is represented by a $k$-dimensional vector, and

- the standard deviation of each linear combination is equal to the length of the corresponding linear combination of vectors.

In particular:

- for $k=4$, we can geometrically describe this representation in terms of quaternions [3] $a+b \cdot \mathrm{i}+c \cdot \mathrm{j}+d \cdot \mathrm{k}$, where:

$$
\begin{gathered}
\mathrm{i}^{2}=\mathrm{j}^{2}=\mathrm{k}^{2}=-1, \mathrm{i} \cdot \mathrm{j}=\mathrm{k}, \mathrm{j} \cdot \mathrm{k}=\mathrm{i}, \mathrm{k} \cdot \mathrm{i}=\mathrm{j}, \\
\mathrm{j} \cdot \mathrm{i}=-\mathrm{k}, \mathrm{k} \cdot \mathrm{j}=-\mathrm{i}, \mathrm{i} \cdot \mathrm{k}=-\mathrm{j} ;
\end{gathered}
$$

- for $k=8$, we can represent it in terms of octonions [3], etc.

Similar representations are possible for multi-D generalizations of complex-valued fuzzy logic.

\section{Acknowledgments}

This work was supported by the Project AI \& Reasoning CZ.02.1.01/0.0/0.0/15003/0000466 and the European Regional Development Fund. It was also supported in part by the US National Science Foundation grant HRD-1242122 (Cyber-ShARE Center). This work was performed when M. Svítek was a Visiting Professor at the University of Texas at El Paso.

The authors are thankful to Vladimir Marik and Hung T. Nguyen for their support and valuable discussions.

\section{References}

1. B. E. Baaquie, Quantum Finance: Path Integrals and Hamiltonians for Options and Interest Rates, Camridge University Press, New York, 2004. 
2. R. Belohlavek, J. W. Dauben, and G. J. Klir, Fuzzy Logic and Mathematics: A Historical Perspective, Oxford University Press, New York, 2017.

3. J. H. Conway and D. A. Smith, On Quaternions and Octonions: Their Geometry, Arithmetic, and Symmetry, A. K. Peters, Natick, Massachusetts, 2003.

4. S. Dick, "Towards complex fuzzy logic, IEEE Transactions on Fuzzy Systems, 2005, Vol. 13, No. 3, pp. 405-414.

5. E. Haven abnd A. Khrennikov, Quantum Social Science, Cambridge University Press, Cambridge, UK, 2013.

6. G. Klir and B. Yuan, Fuzzy Sets and Fuzzy Logic, Prentice Hall, Upper Saddle River, New Jersey, 1995.

7. O. Kosheleva and V. Kreinovich, "Approximate nature of traditional fuzzy methodology naturally leads to complex-valued fuzzy degrees", Proceedings of the IEEE World Congress on Computational Intelligence WCCI'2014, Beijing, China, July 6-11, 2014.

8. O. Kosheleva, V. Kreinovich, and T. Ngamsantivong, "Why complex-valued fuzzy? Why complex values in general? A computational explanation", Proceedings of the Joint World Congress of the International Fuzzy Systems Association and Annual Conference of the North American Fuzzy Information Processing Society IFSA/NAFIPS'2013, Edmonton, Canada, June 24-28, 2013, pp. 1233-1236.

9. V. Kreinovich, H. T. Nguyen, and S. Sriboonchitta, "Quantum Ideas in Economics Beyond Quantum Econometrics", In: Ly H. Anh, Le Si Dong, V. kreinovich, and Nguyen Ngoc Thach (eds.), Econometrics for Financial Applications, Springer Verlag, Cham, Switzerland, 2018, pp. $146-151$.

10. J. M. Mendel, Uncertain Rule-Based Fuzzy Systems: Introduction and New Directions, Springer, Cham, Switzerland, 2017.

11. H. T. Nguyen, V. Kreinovich, and V. Shekhter, "On the possibility of using complex values in fuzzy logic for representing inconsistencies", International Journal of Intelligent Systems, 1998, Vol. 13, No. 8, pp. 683-714.

12. H. T. Nguyen and E. A. Walker, A First Course in Fuzzy Logic, Chapman and Hall/CRC, Boca Raton, Florida, 2006.

13. V. Novák, I. Perfilieva, and J. Močkoř, Mathematical Principles of Fuzzy Logic, Kluwer, Boston, Dordrecht, 1999.

14. C. Servin, V. Kreinovich, and O. Kosheleva, "From 1-D to 2-D fuzzy: a proof that intervalvalued and complex-valued are the only distributive options", Proceedings of the Annual Conference of the North American Fuzzy Information Processing Society NAFIPS'2015 and 5th World Conference on Soft Computing, Redmond, Washington, August 17-19, 2015.

15. D. J. Sheskin, Handbook of Parametric and Nonparametric Statistical Procedures, Chapman and Hall/CRC, Boca Raton, Florida, 2011.

16. M. Svítek, Quantum System Theory: Principles and Applications, VDM Verlag, Saarbrucken, Germany, 2010.

17. M. Svítek, "Towards complex system theory", Neural Networl World, 2015, Vol. 15, No. 1, pp. 5-33.

18. L. A. Zadeh, "Fuzzy sets", Information and Control, 1965, Vol. 8, pp. 338-353. 